\title{
Die Anfänge der Spirometrie im 19. Jahrhundert
}

Meinem Lehrer, Professor Heinrich Buess, zum 70. Geburtstag gewidmet

Von René Renggli

Die Spirometrie stellt heute eine wichtige Methode zur Untersuchung der pulmonalen Ventilation dar. Das Spirogramm gibt Aufschluß über die statischen Lungenvolumina (Vitalkapazität und deren Komponenten) und die Ventilationsgrößen (Atemzeitvolumen, Atemgrenzwert und Sekundenkapazität). Die Spirometrie ermöglicht somit die Erfassung restriktiver und obstruktiver Ventilationsstörungen und kann deshalb wesentliche Hinweise zur Ätiologie einer Ateminsuffizienz liefern.

Die Anfänge der Volumetrie der aufgenommenen und abgegebenen Luftmenge reichen in eine Zeit zurück, in der von einer Analyse der Luft noch nicht die Rede sein konnte. Alfonso Borelli (1608-1679), Nachfolger Galileis, der in Pisa als Mathematikprofessor die Physiologie als Teil der Physik behandelte, war der erste, der ein Verfahren begründete, um die Quantität von Luft, die durch einen einzelnen Atemzug aufgenommen wird, zu messen. Im Selbstversuch maß er die Menge der in die Lungen eingeatmeten Luft, und zwar mit einem graduierten Glaszylinder, in den durch die Saugwirkung der Inspiration Seifenlösung hineinaspiriert wurde. Er konnte so das Volumen von 3375 «digiti cubici» einatmen (ca.2250 ccm). Seine Befunde erschienen 1680/81 in seinem Werk «De motu animalium».

Zwei Engländer, James Keill (1671-1721) und James Jurin (1684-1750), beide Vertreter der iatromathematischen Richtung, bemühten sich um die Verbesserung der Methode Borellis. Sie führten genaue Volumenmessungen der ausgeatmeten Luft durch. Keill schätzte die Zahl der Lungenbläschen auf mehr als $1 \frac{1}{2}$ Milliarden und ihre Oberfläche auf ca.22000 QuadratUnzen (1 Unze = 2,54 cm). Es folgte Stephen Hales, der 1733 ähnliche Werte beschrieb, während Johann Nathanael Lieberkühn (1711-1756) auf bedeutend höhere Zahlen kam als sein Vorgänger. Eine rationelle Volumetrie war jedoch noch nicht möglich, da die Luft in ihrer Zusammensetzung nicht bekannt war und da über den Nutzen der Respiration noch viel Unklarheit herrschte. Auf die Geschichte der Respirationstheorien kann hier nicht genauer eingegangen werden, da es den Rahmen dieser Arbeit sprengen würde. Es sei nur erwähnt, daß durch Black, Rutherford, Lavoisier, 
Priestley und Scheele, um nur die wichtigsten zu nennen, die maßgeblich an der Entdeckung der Zusammensetzung der gebundenen und atmosphärischen Luft mitgearbeitet haben, die Atmungsphysiologie einen großen Schritt weiterkam.

Doch kehren wir zurück zur Volumetrie. Um 1800 waren es Goodwyn, Davy, Thompson, Kentish, Abernethy, Menziès, Kite, Allen und Pepys, Seguin, Herbst und andere, welche die Lungenvolumina zu ermitteln versuchten. Doch keiner der obenerwähnten Beobachter bediente sich zweckmäßiger Instrumente, und keiner fand eine sichere Basis, von der aus die Resultate zuverlässig beurteilt werden konnten. Abernethy zum Beispiel gebrauchte ein weites mit Wasser gefülltes Glas, das er in eine pneumatische Wanne umstürzte und in das er durch eine gekrümmte Röhre alle Luft nach einer tiefen Inspiration ausatmen ließ. Vor Abernethy verwendete Kentish hierzu eine graduierte Glocke. Sein um 1814 konstruiertes Meßgerät nannte er Pulmometer. 1845 erschien dann Karl Vierordts Buch «Physiologie des Athmens mit besonderer Rücksicht auf die Ausscheidung der Kohlensäure». Vierordt zog die Messung der Atmungsgrößen zur Bestimmung des Gaswechsels heran. Hier interessiert jedoch nur seine Volumetrie. Zur Ansammlung der ausgeatmeten Luft konstruierte er einen (wie er ihn nannte) Exspirator. Dies war ein gläserner Ballon mit einem Rauminhalt von 9200

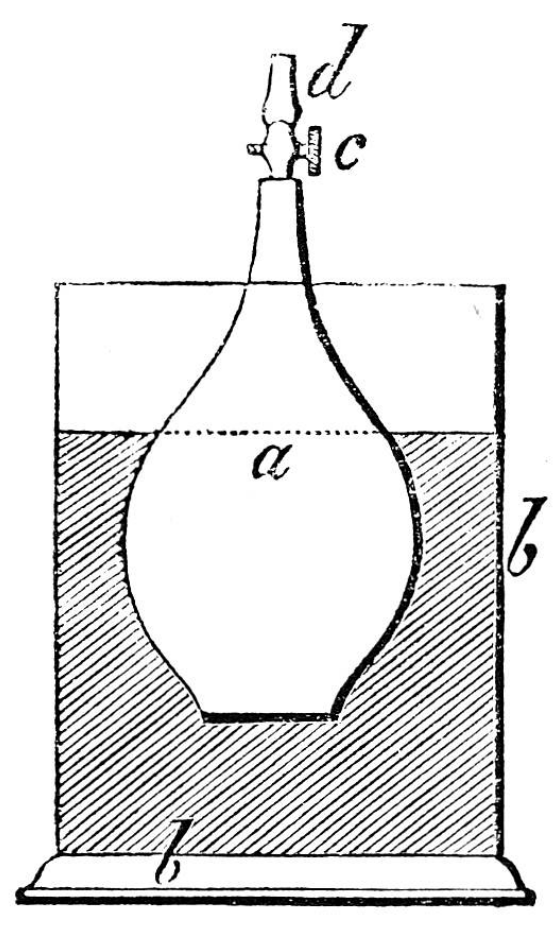

Abb. 1. Exspirator von Karl Vierordt 1845 (ein unten offener Glasballon in Kochsalzlösung) 
ccm, der in einen mit Kochsalzlösung gefüllten Behälter gestellt wurde. Der Glasballon war unten offen, so daß sich eine Art kommunizierendes Gefäß bildete (siehe Abb.1). Gemessen wurde folgendermaßen: Der Proband exspirierte in die Mundöffnung; dabei floß das entsprechende Volumen Kochsalzlösung durch die untere Öffnung aus dem Glasballon, und man konnte das Exspirationsvolumen am Lösungspegel des Glasballons ablesen. Es sei hier nochmals erwähnt, daß Vierordt seinen Apparat vor allem zur Bestimmung der Kohlensäure benutzte. D. h. es ging ihm nicht darum, einen einzelnen Atemstoß zu messen, sondern er ließ meist den ganzen Ballon mit Luft füllen, um die Kohlensäurekonzentration zu messen (seine Werte bewegten sich um $4 \%$ herum). Interessant ist, daß man die heute gemessenen Volumina schon damals kannte. Einzig die Namengebung hat sich geändert. Das heutige Atemzugvolumen entspricht der damaligen Atmungsluft, das exspiratorische Reservevolumen der zurückbehaltenen Luft, das inspiratorische Reservevolumen der Ergänzungsluft und das Residualvolumen der rückständigen Luft. Das vitale Atmungsvermögen kann der heutigen Vitalkapazität gleichgesetzt werden. Wie schon oben erwähnt, variierten die entsprechenden Meßgrößen der verschiedenen Forscher enorm. Und zwar wurden bis 1845 folgende Werte gefunden:

Rückständige Luft:

40 bis $260 \mathrm{KZ}$

Zurückbehaltene Luft:

77 bis $170 \mathrm{KZ}$

Atmungsluft:

3 bis $100 \mathrm{KZ}$

Ergänzungsluft: 119 bis $200 \mathrm{KZ}$

Vitales Atmungsvermögen: 100 bis $300 \mathrm{KZ}$, wobei 1 Kubikzoll (KZ) 16,4 Kubikcentimetern entspricht.

Am 28. April 1846 war dann die Geburtsstunde des Spirometers gekommen. In der damaligen Sitzung der Londoner Ärztegesellschaft verlas deren Sekretär, Dr.George Gursham, die epochemachende Schrift von John Hutchinson mit dem Titel "On the capacity of the lungs, and on the respiratory functions, with a view of establishing a precise and easy method of detecting diseases by the spirometer". Bald darauf wurde die vielbeachtete Schrift im 29. Band der «Medical-chirurgical Transactions» gedruckt (1846) und fand seitdem ungewöhnliche Aufmerksamkeit unter den Ärzten und Physiologen. John Hutchinson, Vater und Erfinder des Spirometers, wurde 1811 in Newcastle-upon-Tyne geboren. Nach dem Studium am University College in London war er einige Zeit Assistant Physician des Hospital for Consumption in Brompton und einer der Physicians der 
Britannia Life Assurance Company. Er machte sich durch seine Studien über die Physiologie der Respiration sehr verdient und war ein sehr erfindungsreicher, für Kunst und Wissenschaft hochbegabter Mann. 1852 verließ er England und lebte bis März 1861 in Victoria und von da an auf den Fidschi-Inseln, von wo er wieder nach England zurückkehren wollte, als er im Juli 1861 plötzlich starb.

Im Januar 1849 erschien die deutsche Fassung von Hutchinsons Schrift, verfaßt von Dr.Samosch. Der erste jedoch, der in Deutschland mit dem Spirometer arbeitete, war Prof. Julius Vogel in Gießen. Er konstruierte ein leicht modifiziertes Gerät, mit dem sein Schüler Gustav Simon Kontrollversuche durchführte. 1848 wurden die Resultate dieser Arbeit als Dissertation Simons mit dem Titel «Über die Menge der ausgeatmeten Luft bei verschiedenen Menschen und ihre Messung durch das Spirometer. Ein Beitrag zur medizinischen Diagnostik» veröffentlicht.

Indessen versuchte Hutchinson, seine Erfindung praktischen Zwecken zuzuführen. In England und auf dem Kontinent wurden in jener Zeit die ersten Lebensversicherungen abgeschlossen. Dabei war es für die Versicherungsinstitute von großer Wichtigkeit, über den Gesundheitszustand der Klienten Bescheid zu wissen. Was lag näher, als das neuerfundene Spirometer als Gradmesser für die Gesundheit einzusetzen, konnten doch auch medizinische Laien ohne große Vorkenntnisse mit dem Spirometer messen. Hutchinson wie auch Simon schließen ihr Werk mit folgenden Worten: «Hiermit sei den Lebensversicherungsanstalten das vorliegende Werk dringend ans Herz gelegt.» Doch zum Nachteil der Medizin und zuungunsten der Versicherungen zeigte sich einige Jahre später, daß der Diagnostik von Lungenkrankheiten durch das Spirometer Grenzen gesetzt sind. Man hatte zuerst gehofft, mit der Spirometrie ein Verfahren gefunden zu haben, um die damals grassierende Lungenschwindsucht oder Phthisis, wie sie auch genannt wurde, zu diagnostizieren. Die große Hoffnung, die man in das neue Verfahren setzte, ist verständlich, wenn man weiß, daß in jener Zeit die Phthisis die Krankheit mit der höchsten Mortalität war. Es seien einige Zahlen aus der Zeit um 1845 genannt. Unter 1000 Sterbefällen waren in München 107, in Paris 200, in London 236 und in Wien 114 Schwindsuchtopfer. Simon schreibt dazu: «Also da, wo uns Perkussion und Auskultation im Stiche lassen, bei chronisch verlaufenden Miliartuberkeln (denn die Akuten sind meist schnell tödlich), ist das Spirometer zur Diagnose derselben das einzige, und seinem Zweck vollkommen entsprechende Mittel, wie aus den schlagenden, von Hutchinson angeführten Fällen ersichtlich ist.» 
Weiter zieht Simon folgende Schlüsse aus seinen und Hutchinsons Beobachtungen: 1. Die Respirationsgröße steht in direktem Verhältnis zur Größe eines Individuums. 2. Bei geringer Beweglichkeit der Brust ist auch die Respirationsgröße vermindert. 3. Ein tuberkulöser Habitus, d.h. eine abgeflachte Brust von geringem Umfang berechtigt nicht zur Voraussetzung von Tuberkulose: Nur das Spirometer ist, wenn Auskultation und Perkussion uns keine Auskunft geben, imstande, darüber zu entscheiden. 4. Miliartuberkeln sind anzunehmen, wenn die Respirationsgröße $1000 \mathrm{ccm}$ geringer ist als die normale, und wenn Auskultation und Perkussion keine objektiven Symptome anzeigen. 5. Bei den übrigen Brustkrankheiten ist das Spirometer nur in prognostischer Beziehung wichtig.

Das erste Hutchinsonsche Spirometer besteht aus einem blechernen, mit einem Luftrohr versehenen Mantel (siehe Abb.2: 1), der mit Wasser gefüllt wird, und aus einem graduierten Gasometer (2), das leicht beweglich in der Wassersäule innerhalb des Mantels aufgehängt ist. Der Mantel hat eine Höhe von $32 \mathrm{~cm}$ und einen Durchmesser von $22 \mathrm{~cm}$. Am Boden besitzt er einen Hahn (3) zum Ablassen des Wassers. Am oberen Rande des Mantels ist hinter und über einem beweglichen Index (4) ein Glasstreifen (5) eingekittet, durch den man den Wasserstand im Mantel ablesen kann. Am oberen Rande sind zwei Hülsen (6) angebracht, in die zwei durch einen Querbalken (8) verbundene Stangen eingesteckt werden. Links und rechts des Querbalkens befinden sich Rollen (9), über welche seidene Schnüre führen, die einerseits am Deckel des Rezipienten und andererseits an Gewichten (10) befestigt sind. Das Gewicht ist so ausbalanciert, daß schon ein schwacher Hauch genügt, um das Gasometer in der Wassersäule zu heben. Außen am Mantel führt ein Inspirationsrohr (11), das am zweiten Viertel der Mantelhöhe beginnt, abwärts, geht am Boden durch den Mantel hindurch bis in die Mitte und erhebt sich dann senkrecht bis zum oberen Rand. Das Luftrohr besitzt in der Mitte der Mantelhöhe einen Hahn (12) und unter diesem einen Schenkel (13), der bis zum oberen Rand reicht und hier nach doppelter Biegung in einem kurzen, abwärtsgerichteten Schenkel ausläuft. In diesem wird ein mit Wasser oder Quecksilber gefülltes Manometer aus Glas (14) eingefügt. An die Mündung des Luftrohrs wird eine vulkanisierte Kautschukröhre (15) mit einem Mundstück luftdicht befestigt. Das Luftrohr kann unten noch mit einem eigenen Abzugshahn (16) versehen werden, um das Wasser abzulassen, das durch eine Person, die nicht ausatmet, sondern einatmet, in das Rohr gezogen wird. Das Gasometer oder der Rezipient hat in seiner Decke eine weite Öffnung (17) zum Auslassen der Luft, die durch ein 
Ventil (18) verschlossen werden kann. An beiden Seiten ist das Gasometer mit Ringen (19) versehen, die beim Steigen des Gasometers durch Anfüllung mit Luft die Leitung in den senkrechten Stangen gewährleisten. Am vorderen Rand befindet sich eine dünne, metallene Skala (20), die leicht beweglich an einem senkrechten Draht verläuft.
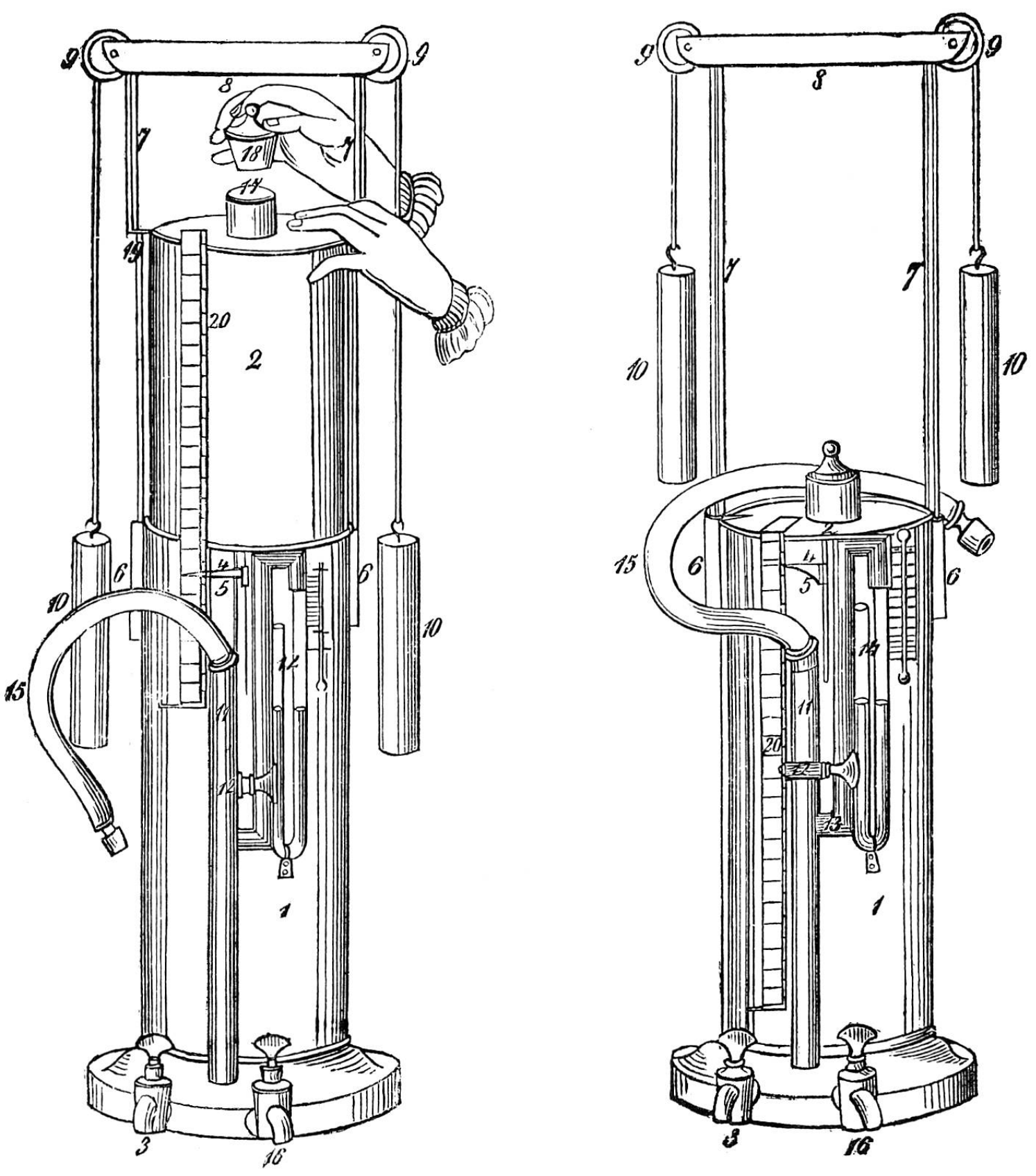

Abb.2. Spirometer von John Hutchinson 1846 
Nach Hutchinson waren es, um nur die wichtigsten zu nennen, in Deutschland Vogel und sein Schüler Simon, dann Haeser, Arnold, Albers, Stellwag, Küchenmeister und Wintrich, in Holland Fabius und Schneevogt, in England Davies, Green, Walshe und Pereira und in Frankreich Hecht, die sich mit der Weiterentwicklung der Spirometrie beschäftigten. Es wurde versucht, das doch recht komplizierte Gerät von Hutchinson zu vereinfachen. Vogel, Stellwag u. a. arbeiteten mit einem solchen modifizierten Gerät, das aber den Nachteil hatte, daß man zwar sehen konnte, wie hoch der Wasserspiegel im äußeren Mantel stand, aber nicht, wie hoch er im Gasometer selbst war, da dieser aus Blech gefertigt wurde. 1854 war es dann M. A. Wintrich, der ein modifiziertes, besseres Gerät (siehe Abb.3) konstruierte, bei dem die Wasserspiegel gut ablesbar waren. Er verwendete dazu eine Glasglocke und kittete außerhalb des Spirometers einen Glasstreifen ein, damit die beiden Wasserpegel gut sichtbar waren. Die Glocke faßt 5500 ccm Luft. Ihr Kubikinhalt ist durch Teilstriche von je $20 \mathrm{ccm}$ unterteilt. Oben läuft die Glocke schmal aus und enthält dort ein Thermometer. Auf die auslaufende Röhre ist eine messingene Hülse mit zwei Hähnen gekittet, um das Spirometer zur Untersuchung der Atmungsluft und zur Bestimmung der Lungenelastizität benützen zu können. Mit diesem Gerät untersuchte Wintrich 4000 Personen, worunter 500 Kranke waren. Hutchinson hatte in seiner Arbeit nur 60 Kranke und insgesamt 2130 Personen untersucht. Als maßgebende Verhältnisse für das vitale Atmungsvermögen fand Wintrich folgende drei Größen: 1. die Größe und Höhe des Körpers und dessen Stellung, 2. das Gewicht, 3. das Alter. Nicht von Wichtigkeit seien, wie Hutchinson noch gemeint hatte, die Geräumigkeit des Brustkorbs, der Brustumfang und die Beweglichkeit desselben. Wintrich schreibt: «Kinder haben nach meinen Untersuchungen eine größere Beweglichkeit des Brustkastens als Erwachsene und doch ein bedeutend kleineres Atmungsvermögen.»

1855 widmete Friedrich Arnold in seinem Buch «Ueber die Athmungsgröße des Menschen» dem Wert der Spirometrie ein eigenes Kapitel, das hier als repräsentativ für jene Zeit kommentiert sei. Arnold meint, in diagnostischer Hinsicht sei das Spirometer von besonderem Wert bei der chronischen Miliartuberkulose, welche durch die übrigen diagnostischen Hilfsmittel nicht oder zu spät erkannt würde, so daß die geeigneten medizinischen oder diätetischen Hilfsmittel häufig nicht zu einer Zeit angewendet werden, in der noch Hilfe durch Änderung des Aufenthaltsortes, Wechsel des Berufs, geeignete Übung der Atmungsorgane usw. möglich wäre. Außerdem scheine 


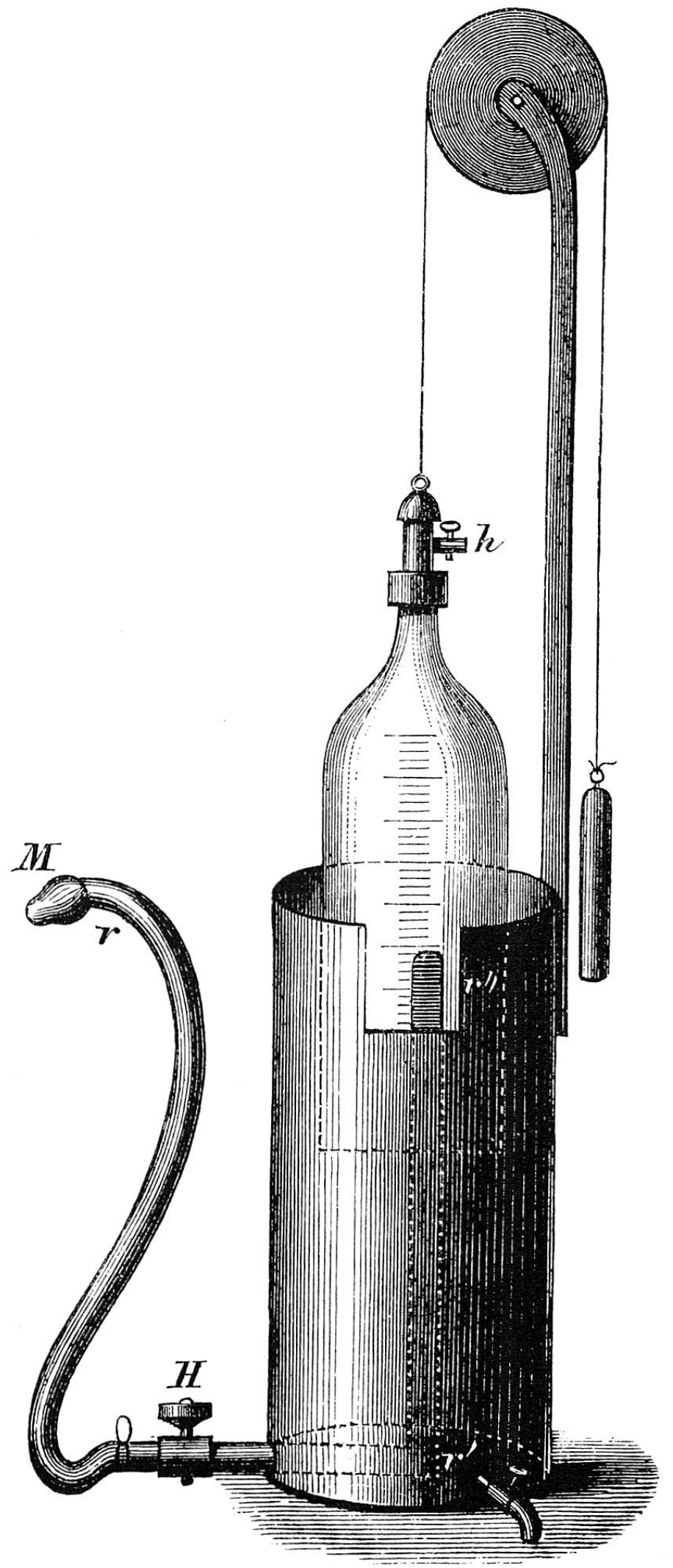

Abb. 3. Spirometer nach Anton Wintrich 1854

\section{4}


die Spirometrie auch bei der Diagnose des Lungenemphysems von Nutzen sowie bei fettreichen Thoraxwänden, bei denen Perkussion und Auskultation erschwert seien. Der prognostische Wert stütze sich auf die Wegsamkeit der Lungen und somit auf die vitale Kapazität, woraus man auf Grund der Ausbreitung der Tuberkulose, der Hepatisation in der Pneumonie, der Veränderung des Lungengewebes als Folge einer chronischen Bronchitis und auf die Größe eines pleuritischen Exsudats schließen könne. Von besonderer Bedeutung sei das Spirometer aber in prophylaktischer Hinsicht. Bei Personen, deren Angehörige an Phthisis starben oder bei denen eine erbliche Anlage vorausgesetzt werden könne, ohne daß aber Perkussion und Auskultation ein Resultat ergeben hätten, sei das Spirometer ebenfalls anzuwenden. Bei scheinbarer Gesundheit liegen die Untersuchungsresultate solcher Personen 500-1000 ccm unter dem physiologischen Mittel. Auch bei der Untersuchung von Konskriptionspflichtigen, bei Berufswahl und Lebensversicherungen sei das Spirometer dienlich. Keine Anwendung finde es in allen Fällen von Lungenkrankheiten, die mit Fieber oder heftigen Schmerzen verbunden sind. Dann nämlich könne nicht genügend tief inspiriert werden. Ebensowenig sei das Spirometer bei asthmatischen Anfällen, heftigen Atemkrämpfen und großer Schwäche der Kranken anzuwenden.

In der Zeit bis zum Ende des 19. Jahrhunderts gab es keine tiefgreifenden Veränderungen mehr. Es wurde lediglich langsam klar, daß man übertriebene Erwartungen in die Spirometrie gesetzt hatte, und zwar besonders in bezug auf die Diagnose der Phthisis. Willibald Nagel schreibt zu Beginn des 20. Jahrhunderts: «Es versteht sich, daß ausgedehnte Atelektasen, Emphyseme oder Infiltrationen die Vitalkapazität herabsetzen werden, indessen wird bei den großen individuellen Verschiedenheiten eine solche Veränderung nur bei Untersuchung desselben Menschen im gesunden und später im kranken Zustande erkennbar sein. Da ferner die geringen Veränderungen im Beginne der Phthisis den in Rede stehenden Wert noch gar nicht beeinflussen, kann von einer diagnostischen Bedeutung der Spirometrie heutzutage kaum mehr die Rede sein.»

Jedoch eine Neuerung technischer Natur sei noch erwähnt. Peter Ludwig Panum war wohl der erste, der die Lungenvolumina graphisch darstellte, und zwar durch Anbringen eines Schreibstifts am Spirometer, der auf einer horizontalen Fläche die entsprechenden Volumina auftrug. Johannes Gad war es dann, der diese Technik verfeinerte (siehe Abb.4). Mit der Konstruktion seines «Aeroplethysmographen» oder Atemvolumenschreibers (1892), bei dem die Glocke durch einen parallelepipedischen Deckel ersetzt ist, der 


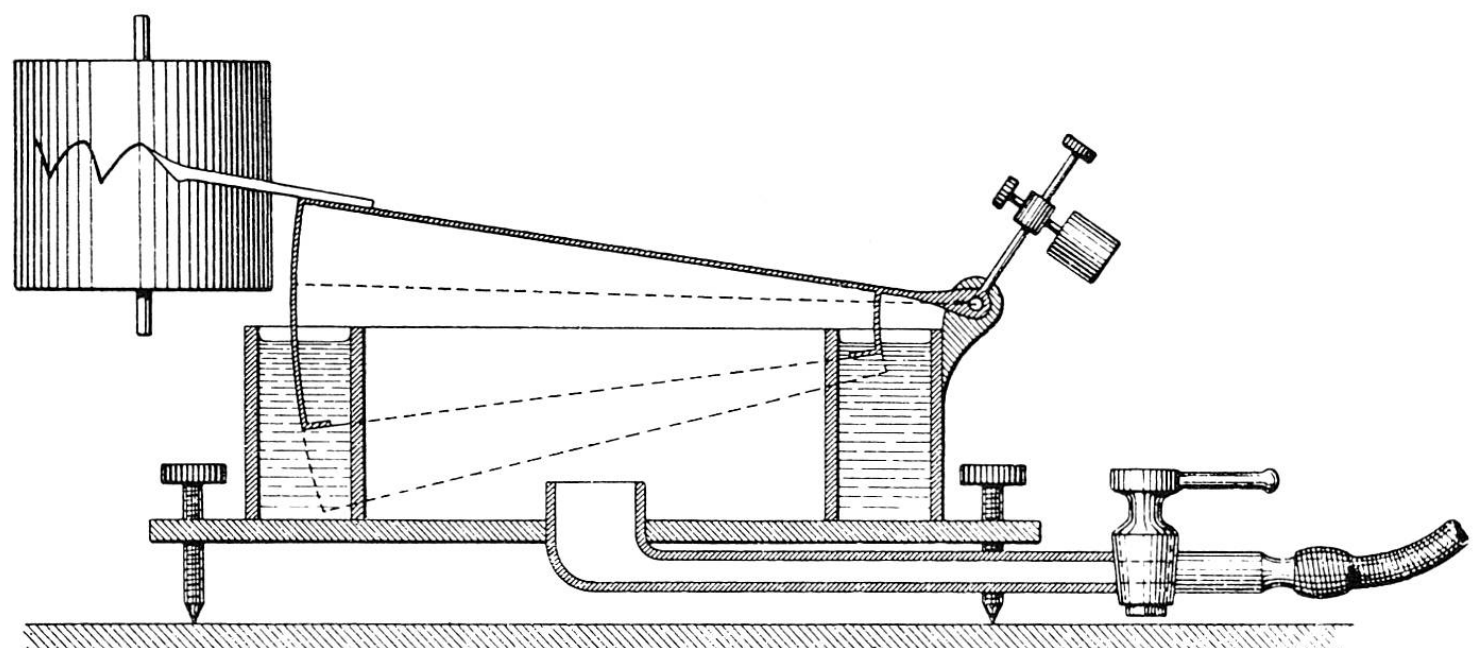

Abb.4. Aeroplethysmograph (Atemvolumenschreiber) von Johannes Gad 1892

sich um eine einer Kante parallele horizontale Achse im Kreisbogen auf- und abbewegt und der mit seinen Rändern in eine am Umfang des unteren Gefäßes angebrachte, zwecks Abdichtung mit Wasser gefüllte Rinne eintaucht, schuf Gad eine Modifikation. Dieser Deckel ist aus sehr leichtem Material gebildet, nämlich aus zweckmäßig dünnem Aluminiumblech, und er ist durch ein senkrecht zur Achse verschiebbares Laufgewicht genau äquilibriert. Bei längeren Registrierungen der Atmung mußte eine große Flasche als Vorlage dazwischengeschaltet werden, deren Luftinhalt öfters erneuert werden mußte, damit eine Dyspnoe des Probanden vermieden wurde. Eine gewisse Trägheit und eine zu große Reibung haftete diesem Apparat jedoch an. Deshalb versuchten in der Folge Lindhagen und in Gads Laboratorium R.F.Fuchs, wieder mit kleinen Glockengasometern die Atemvolumenschreiber zu verbessern.

Zum Schluß sei noch Thomas Gregor Brodie erwähnt, der 1902 eine neue Art der Volumetrie vorstellte. Er konstruierte den ersten Balgenschreiber, d.h. das erste Trockenspirometer. Es ist dies ein Vorläufer des heute verwendeten Spirotests «Metabo» nach Fleisch. Mit dieser Erfindung sind wir eigentlich, was die Theorie betrifft, auf dem heutigen Stand der Volumetrie angelangt. Einzig in technischer Hinsicht gab es im 20. Jahrhundert noch einige Verbesserungen; aber der Grundstein zur Spirometrie wurde in der zweiten Hälfte des 19. Jahrhunderts gelegt. 


\section{Zusammenfassung}

Als Vater der Spirometrie darf man John Hutchinson ansehen; doch hat bereits Borelli zu Beginn des 17. Jahrhunderts eine primitive Volumetrie der eingeatmeten Luft vorgenommen. Nach ihm waren es Kentish mit seinem Pulmometer, Allen und Pepys, Vierordt und andere, die versuchten, die Volumina zu bestimmen. Jedoch fand keiner eine sichere Basis, von welcher aus die Resultate zuverlässig beurteilt werden konnten. Nachdem dann Hutchinson 1846 den Grundstein zur Spirometrie gelegt hatte, waren es Vogel, Simon und Wintrich, um nur ein paar zu nennen, die technische Verfeinerungen anbrachten. Um 1900 waren es Panum und etwas später Gad, die mittels eines Schreibers die Volumina zum erstenmal graphisch darstellten. Endlich entwickelte Brodie 1902 den ersten Balgenschreiber, womit die Volumetrie, was die Theorie betrifft, auf dem heutigen Stand angelangt war.

\section{Literatur}

Allen, W., und Pepys, W. H., On the changes produced in the atmospheric and oxygen gas by respiration. Phil. Trans. R. Soc., 1808.

Arnold, F., Über die Atmungsgröße des Menschen, Heidelberg 1855.

Berzelius, J. J., Lehrbuch der Chemie, 2. Aufl., Band 4: Thierchemie, Dresden 1833.

Borelli, G. A., De motu animalium, Leiden 1710.

Borutteau, H., Versuch einer kritischen Geschichte der Atmungstheorien. Arch. Gesch. Med.2, 1909.

Brodie, T.G., On recording variations in volumetry by airtransmission. J. Physiol. (Lond.) $27,1902$.

Buess, H., Zur Geschichte der Atmungslehre und Zeittafel zur Entwicklung der Atmungsphysiologie von Lavoisier bis zum Ende des 19. Jahrhunderts. Ciba Zeitschr.91, 1943.

Fleisch, A., Spirotest. Schweiz. med. Wschr.26, 1965.

Gad, J., Die Regulation der normalen Atmung. Arch. Physiol., 1880.

Günther, A. F., Lehrbuch der Physiologie des Menschen, Band 2, 1. Abt., Leipzig 1848.

Hecht, L. E., Le Spiromètre, Strasbourg 1855.

Hermann, L., Handbuch der Physiologie, Band 4, Teil 2, Leipzig 1882.

Hirsch, A., Biographisches Lexikon der hervorragenden Ärzte aller Zeiten und Völker, Berlin/Wien 1931.

Hutchinson, J., Von der Kapazität der Lungen und von den Atmungsfunktionen, Braunschweig 1849.

Kentish, E., An account of baths ..., with the description of a pulmometer, London 1814.

Liebig, J. von, Die Thier-Chemie oder die anorganische Chemie in ihrer Anwendung auf Pathologie und Physiologie, 2. Aufl., Braunschweig 1843. 
Nagel, W., Handbuch der Physiologie des Menschen, Band 1, Braunschweig 1909.

Pettenkofer, M. von, Über einen neuen Respirationsapparat, München 1861.

Simon, G., Über die Menge der ausgeatmeten Luft bei verschiedenen Menschen und ihre Messung durch das Spirometer, Diss. Gießen 1848.

Speck, C., Physiologie des menschlichen Atmens, Leipzig 1892.

Vierordt, K., Physiologie des Atmens, Karlsruhe 1845.

Voigt, C., Beschreibung eines Apparates zur Untersuchung der gasförmigen Ausscheidung des Thierkörpers. Math. Phys. Abhandl. K. Bayr. Akad., Band 12, München 1876.

Wintrich, M.A., Handbuch der speziellen Pathologie und Therapie, Band 5, I.Abt.: Die Krankheiten der Respirationsorgane, hrsg. von R. Virchow, Erlangen 1854.

Zuntz, N., und Schumburg, W.A.E.F., Studien zu einer Physiologie des Marsches, Berlin 1901.

\section{Summary}

At the beginning of the 17th century, Borelli built a first type of a spirometer. After him it was Kentish with his pulmometer, Allen and Pepys, Vierordt and others who tried to determine the volumes of the lungs. In 1846, John Hutchinson laid the foundation-stone of modern spirometry. Vogel, Simon, Wintrich and others made refinements. Around 1900, Panum, and later Gad constructed the first volume writer. Finally it was Brodie who built a first bellows writer.

Dr. med. René Renggli

Physiologisches Institut der Universität Basel

Vesalgasse 1

CH-4051 Basel 